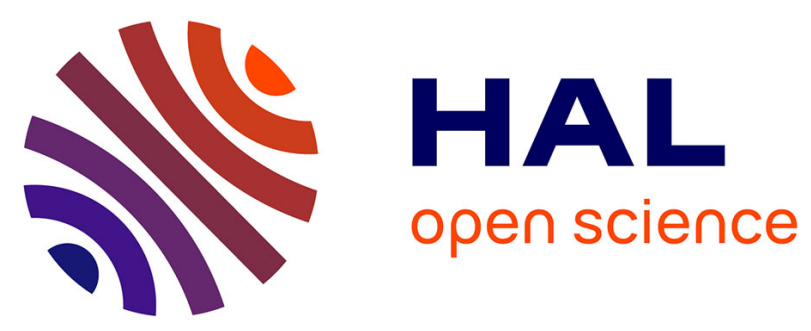

\title{
Three-dimensional Simulations and Spacecraft Observations of Sub-ion Scale Turbulence in the Solar Wind: Influence of Landau Damping
}

Sumire Kobayashi, Fouad Sahraoui, T. Passot, D. Laveder, P. Sulem, S. Y. Huang, Pierre Henri, R. Smets

\section{To cite this version:}

Sumire Kobayashi, Fouad Sahraoui, T. Passot, D. Laveder, P. Sulem, et al.. Three-dimensional Simulations and Spacecraft Observations of Sub-ion Scale Turbulence in the Solar Wind: Influence of Landau Damping. The Astrophysical Journal, 2017, 839 (2), pp.122. 10.3847/1538-4357/aa67f2 . insu-01670788

\section{HAL Id: insu-01670788 \\ https://hal-insu.archives-ouvertes.fr/insu-01670788}

Submitted on 20 Dec 2018

HAL is a multi-disciplinary open access archive for the deposit and dissemination of scientific research documents, whether they are published or not. The documents may come from teaching and research institutions in France or abroad, or from public or private research centers.
L'archive ouverte pluridisciplinaire HAL, est destinée au dépôt et à la diffusion de documents scientifiques de niveau recherche, publiés ou non, émanant des établissements d'enseignement et de recherche français ou étrangers, des laboratoires publics ou privés. 


\title{
Three-dimensional Simulations and Spacecraft Observations of Sub-ion Scale Turbulence in the Solar Wind: Influence of Landau Damping
}

\author{
S. Kobayashi ${ }^{1}$, F. Sahraoui ${ }^{1}$, T. Passot ${ }^{2}$, D. Laveder $^{2}$, P. L. Sulem ${ }^{2}$, S. Y. Huang ${ }^{3}$, P. Henri ${ }^{4}$, and R. Smets ${ }^{2}$ \\ ${ }^{1}$ Laboratoire de Physique des Plasmas, CNRS-Ecole Polytechnique-UPMC-Univ. Paris-Sud-Observatoire de Paris, \\ Route de Saclay, F-91128, Palaiseau, France; sumire.kobayashi@1pp.polytechnique.fr \\ ${ }^{2}$ Université Côte d'Azur, CNRS, Observatoire de la Côte d'Azur, Laboratoire J.L. Lagrange, \\ Boulevard de l'Observatoire, CS 34229, F-06304 Nice CEDEX 4, France \\ ${ }^{3}$ School of Electronic Information, Wuhan University, Wuhan, China \\ ${ }^{4}$ Laboratoire de Physique et Chimie de l'Environnement et de l'Espace, CNRS, Orléans, France \\ Received 2017 February 2; revised 2017 March 9; accepted 2017 March 10; published 2017 April 24
}

\begin{abstract}
Three-dimensional nonlinear finite Larmor radius (FLR)-Landau fluid simulations, which include some smallscale $\left(k_{\perp} \rho_{i} \gtrsim 1\right)$ kinetic effects, are performed to explore the nature of the sub-ion scale turbulence in the solar wind and to investigate the role of Landau damping and FLR corrections. The resulting steady-state magnetic power spectrum in the dispersive range display exponents that vary within a range of values compatible with statistical results reported from in situ spacecraft measurements of solar wind turbulence as well as from gyrokinetic simulations. The spectral slopes are shown to depend on the strength of the nonlinear effects and on the scale at which turbulent fluctuations are driven in the simulations. The influence of Landau damping is addressed by comparison with simulations where the double-adiabatic closure is imposed. The role of FLR corrections is also analyzed. Comparison with in situ observations in the solar wind are performed to enlighten the influence of the fluctuations power at different scales on the spectral slopes in the sub-ion range. Using diagnosis of both magnetic compressibility and frequency-wavenumber spectra, it is shown that in spite of the evidence of the presence of fastmagnetosonic modes, the magnetic energy is mostly distributed around the kinetic Alfvén waves and the slow modes, in agreement with solar wind measurements. The observed large broadening about the linear dispersion relations may reflect the presence of coherent structures.
\end{abstract}

Key words: acceleration of particles - magnetic fields - magnetohydrodynamics (MHD) - plasmas - solar wind turbulence

\section{Introduction}

Many laboratory and astrophysical plasmas such as the solar wind, the Earth's magnetosheath, and fusion devices are only weakly collisional. In those plasmas, understanding turbulent heating and acceleration of particles depends critically on the nature of the processes that dissipate electric and magnetic fluctuations at kinetic scales, $k \rho_{i} \gtrsim 1$ (Schekochihin et al. 2009). However, the physics underlying those critical scales are not fully understood and remain subject to strong controversies.

The solar wind provides a unique opportunity to study collisionless plasma turbulence thanks to the availability of high quality in situ data from several operating spacecraft, such us WIND, STEREO, Cluster, or Themis. Decades of observational studies have shown a $f^{-5 / 3}$ dependence of the power spectrum density (PSD) of the magnetic field fluctuations in the frequency range $\sim\left[10^{-4}, 10^{-1}\right] \mathrm{Hz}$, generally interpreted as evidence of the turbulent energy cascade in the inertial range. These observations are consistent with strong Alfvénic turbulence theory derived in the MHD limit (see review in Bruno \& Carbone 2005). When the energy cascade reaches the ion scale, kinetic effects become important and are thought to play a significant role in dissipating turbulence energy into plasma heating. Indeed, the PSD of the magnetic fluctuations measured in the solar wind show clear steepening to $f^{-\alpha}$, where $\alpha \sim[2.3,5]$, near the ion scale (Goldstein et al. 1994; Leamon et al. 1998b; Smith et al. 2006; Sahraoui et al. 2010). That steepening has generally been attributed to an enhancement of dissipation of the magnetic energy. However, the actual dissipation mechanisms remain unclear, and different processes have been proposed, such as cyclotron or Landau damping of Alfvenic fluctuations (Goldstein et al. 1994; Leamon et al. 1998a, 1999; Marsch 2006; Sahraoui et al. 2010); local plasma instabilities generated by, for example, temperature anisotropy (Bale et al. 2009); or dissipation within reconnecting current sheets (Markovskii \& Vasquez 2011; Huang et al. 2016). Using high time resolution of the Cluster/ STAFF instrument that allows one to access higher frequencies of the spectrum, Sahraoui et al. (2010) showed that the steepening to $\sim f^{-4}$ is actually limited to a narrow scale range $k_{\perp} \rho_{i} \sim[0.5,3]$, which was referred to as the transition range, and that the spectrum flattens at smaller scales. Note that in some cases, the transition range does not exist. At scales smaller than the ion gyroscale and extending down to the electron gyroscale, statistical studies using the Cluster/STAFF observations showed that the PSD of the magnetic fluctuations follow power-law-like scaling, with an exponent covering the range $\sim[-2.5,-3.1]$ and a peak near -2.8 (Alexandrova et al. 2012; Sahraoui et al. 2013). A similar distribution of slopes at the sub-ion scales has been reported in planetary magnetosheath turbulence (Huang et al. 2014; Hadid et al. 2015), despite the differences in the plasma parameters in the two regions (e.g., the plasma $\beta$ and temperature anisotropy) and even when an inertial range has not been observed at the MHD scales (Hadid et al. 2015; Huang et al. 2017a). This may suggest a possible universal-like distribution of sub-ion scales spectral slopes, although the spread in the values of the exponents is larger than that reported in the inertial range (Smith et al. 2006). In contrast, the slopes within the transition 
range seem to depend on the power of the fluctuations in the inertial range and on the speed of the solar wind (slow versus fast) - the higher the power, the steeper the spectrum (Smith et al. 2006; Bruno et al. 2014). A similar dependence of the spectral slopes both above and below the electron scales on the forcing amplitude in the kinetic range has been reported in three-dimensional PIC simulations of whistler turbulence, but in the opposite sense, the higher the forcing amplitude, the shallower the spectrum (Gary et al. 2012). We will return to this point in Section 3.1.

Another still hotly debated question concerns the nature of the plasma modes carrying the energy cascade down to the subion and electron scales. Although there is an increasing body of observational evidence that strongly anisotropic Kinetic Alfvén Wave (KAW) turbulence plays a leading role in that cascade (at least in the solar wind; Bale et al. 2005; Sahraoui et al. 2009, 2010; Podesta \& TenBarge 2012; Chen et al. 2013; Kiyani et al. 2013), other studies suggest that other modes may exist (at least as a minor component of the turbulence), such as whistler or Bernstein modes (Gary et al. 2012; Podesta \& TenBarge 2012; Sahraoui et al. 2012), as already reported in observational studies in the magnetosheath (Sahraoui et al. 2003), in the cusp region (Grison et al. 2005), and in the solar wind (He et al. 2011; Klein et al. 2014).

On the other hand, existing numerical simulations yield very different answers to the question as to which plasma mode (or modes) dominates the cascade at kinetic scales, the main reason being the differences in the numerical setups. Simulations of 3D incompressible Hall-MHD without a guide field showed the coexistence of both whistler (right-handed) and Alfvén (lefthanded) modes with respective magnetic spectra scaling like $k^{-7 / 3}$ and $k^{-11 / 3}$ (Meyrand \& Galtier 2012). However, HallMHD, being a fluid theory, does not contain any kinetic damping, and therefore cannot adequately answer the question of interest here. Three-dimensional PIC simulations (initialized with waves fulfilling linear dispersion relation of whistler modes) showed evidence for the dominance of whistler turbulence at small scales with a transverse magnetic fluctuation spectrum, hereafter denoted $E\left(B_{\perp}\right)$, scaling like $k_{\perp}{ }^{-3.1}$ (Chang et al. 2011; Gary et al. 2012). Two-dimensional implicit PIC simulations, initialized with random fluctuations, showed no clear (linear) plasma mode dominating turbulence at electron scales (Camporeale \& Burgess 2011). A similar conclusion was reached by Parashar et al. (2010), who used 2D hybrid simulations initialized with Orszag-Tang vortex, where dissipation is found to happen via magnetic reconnection occurring within localized current sheets (Chasapis et al. 2015; Huang et al. 2017b). However, 3D PIC simulations driven by shear flow showed the coexistence of current sheets and waves (KAWs and magnetosonic modes), suggesting that dissipation occurs via reconnection and wave-particle interactions (Karimabadi et al. 2013). Three-dimensional gyrokinetic (GK) simulations, valid for low-frequency and strongly anisotropic turbulence $\left(\omega \ll \omega_{c i}\right.$ and $k_{\|} \ll k_{\perp}$; Schekochihin et al. 2009; Tatsuno et al. 2009; Plunk et al. 2010; Howes et al. 2011), which include the physics of Landau damping and finite Larmor radius (FLR) effects, showed a spectrum of transverse magnetic energy $E\left(B_{\perp}\right) \propto k_{\perp}{ }^{-2.8}$ for KAW-dominated turbulence (Howes et al. 2011), where the dominant energy dissipation mechanism is Landau damping (Howes et al. 2011; TenBarge \& Howes 2013). Other GK simulations with scales extending to $k_{\perp} \rho_{i} \gtrsim 50$, and which focus on collisional dissipation effects, display a slightly steeper spectrum (Told et al. 2015). However, since this model does not retain high frequency modes (i.e., fast or whistler modes), it cannot fully answer the question relative to the dominant plasma mode at kinetic scales.

In this article, we use the FLR-Landau fluid (FLR-LF) model (Passot \& Sulem 2007; Passot et al. 2014; Sulem \& Passot 2015), inspired by a Landau fluid model of Snyder et al. (1997; see also references therein) derived from drift kinetics and valid at large scales only. The model is used to investigate solar wind turbulence and compare the obtained results to in situ solar wind observations from the Cluster mission. The FLR-LF model allows us to build bridges between fluid models (e.g., Hall-MHD) that do not contain any kinetic effect, and fully kinetic models that are computationally expensive. Indeed, full kinetic simulations are generally limited to some physical space (e.g., $\omega \ll \omega_{c i}$ and $k_{\|} \ll k_{\perp}$ in GK models), use in some instances an unrealistic proton to electron mass ratio in the range $25-100$, or reduce the dimensionality of the problem (1D or $2 \mathrm{D}$ simulations). The main advantage of the FLR-LF model is that it uses a fluid approach (less computationally expensive than the full kinetic models) while retaining the essential kinetic effects relevant to describe the sub-ion scale physics of the solar wind, namely linear Landau damping and FLR effects. Moreover, the model allows one to reproduce plasma instabilities (e.g., the mirror instability) that may be generated in the plasma by ion temperature anisotropy. These instabilities are frequently observed in the solar wind and magnetosheath (Gary et al. 1993; Kasper et al. 2002; Marsch et al. 2004; Sahraoui et al. 2004, 2006; Hellinger et al. 2006; Matteini et al. 2007; Bale et al. 2009) and may drive different waves in the dissipation range, which in turn can inject energy into the electromagnetic turbulence (Sahraoui et al. 2006; Schekochihin et al. 2009; He et al. 2011; Podesta \& Gary 2011). In order to understand the role of each kinetic effect in damping the turbulent fluctuations and heating the plasma, we consider models with different physical complexities: (i) compressible Hall-MHD with double-adiabatic closure for the dynamical gyrotropic pressure equations (Chew et al. 1956), (ii) Hall-MHD with Landau damping, and (iii) Hall-MHD with Landau damping and FLR corrections (hereafter denoted CGLHMHD, LF, and FLR-LF, respectively). In the following sections, we present the results of those comparisons with the aim of addressing the following specific questions: (i) How do the Landau damping and the FLRs affect the scaling of the turbulent spectra at sub-ion scales? (ii) How are the spectral slopes at the sub-ion scales affected by the fluctuation amplitude at the driving scale? (iii) What is the nature of the plasma modes involved in the cascade at those scales? (iv) What is the nature of the coherent structures that form at kinetic scales?

\section{FLR-LF Simulations}

\subsection{The FLR-LF Model}

The FLR-LF model extends anisotropic compressible HallMHD by including low-frequency linear kinetic physics, namely the Landau damping and FLR effects, which are thought to play a key role in dissipating energy at small scales. Interestingly, the simulations can be executed with varying levels of physics, such as adding and removing Landau damping and FLR effects. This enables us to explore the 
dominant physical mechanisms that are contributing to energy dissipation at kinetic scales. The model is constructed in the following way: the fluid hierarchy for the gyrotropic moments is closed by evaluating the gyrotropic fourth rank cumulants and the non-gyrotropic contributions to all the retained moments, in a way consistent with the linear kinetic theory within low-frequency asymptotics. In brief, the expression of the various moments provided by the low-frequency linear kinetic theory are combined to eliminate the plasma dispersion functions as much as possible, while suitable Pade approximations are used when this is not possible (Passot \& Sulem 2007; Sulem \& Passot 2015). The model reproduces correctly the low-frequency linear kinetic theory at the ion scales (Passot et al. 2012; Hunana et al. 2013). It is also consistent with a weakly nonlinear gyrofluid description (Tassi et al. 2016). It takes into account the electron Landau damping and can be extended down to the electron spatial scales, a situation where electron inertia and electron FLRs would however have to be retained. Unlike the GK approach, the FLR-LF model captures the fast mode dynamics up to the ion cyclotron frequency, although the cyclotron resonance is not described. The model is $3 \mathrm{D}$, which makes it very appropriate to study solar wind turbulence, considering the inherently 3D nature of Alfvénic turbulence (Howes 2015).

Although it is generally admitted that solar wind turbulence in the inertial range is formed by $\sim 90 \%$ of (incompressible) Alfvénic fluctuations and $\sim 10 \%$ of (passively advected) compressible slow modes (Howes et al. 2012), the role of density fluctuations in the turbulence dynamics is far from being negligible. Indeed, compressible fluctuations were shown to increase the energy cascade rate in the inertial range of solar wind and magnetosheath turbulence (Carbone et al. 2009; Banerjee et al. 2016; Hadid et al. 2017; L. Z. Hadid et al. 2017, in preparation), and to modify the scaling of the magnetic energy spectra at smaller scales (Alexandrova et al. 2008). Theoretical study of reduced MHD predicts that slow modes with $k_{\|} \lambda_{m f p} \ll 1$ are effectively undamped ( $\lambda_{m f p}$ is the particle mean free path), and are passively advected by the Alfvén wave turbulence in the inertial range (Schekochihin et al. 2009). Slow modes remain correlated along the field lines in the absence of dissipation in the perpendicular direction. However, at the ion Larmor radius scale and below, Alfvénic fluctuations decouple from the slow modes, and Landau damping is considered to significantly damp slow-mode turbulence linearly (via parallel phase mixing). Previous study with FLR-LF simulations for decaying turbulence found that linear Landau damping is responsible for the significant damping of magnetosonic waves (Hunana et al. 2011).

\subsection{Simulations Setup}

Since solar wind turbulence is generally thought to stem from isotropic random-like fluctuations at scales of the order of (or larger than) the correlation length, it can be justified to utilize random forcing as a turbulence driver when simulating turbulence in the inertial range. In contrast, our study focuses on the turbulence cascade at ion scales, where turbulence becomes strongly anisotropic (i.e., $k_{\perp} \gg k_{\|}$) and dominated by KAWs (Bale et al. 2005; Sahraoui et al. 2010; Podesta \& TenBarge 2012). Therefore we drive our simulations with counter-propagating KAWs that fulfill the linear dispersion relation at the driving scale (chosen to be at the largest scales of the simulation domain). This simulation setup is found to efficiently couple the turbulent fluctuations and generate steady-state turbulent cascade, as shown in GK simulations (Howes et al. 2008, 2011).

In the present simulations, we introduced two thresholds in order to constrain the the sum of kinetic and magnetic energies to stay within a certain range. In the phase of increasing energy, the system is driven until the upper threshold is reached. Afterwards, the energy decreases and the forcing is turned on again only when the lower threshold is crossed. In that way, the forcing is not too impulsive. It turns out that in the stationary regime, the forcing acts approximately $10 \%$ of the time of the simulation. As a driving force we use

$$
F_{i}(t, x)=\sum_{1<n<N} F_{i, n}^{0} \cos \left(\omega_{\mathrm{KAW}}\left(\boldsymbol{k}_{n}\right) t-\boldsymbol{k}_{n} \cdot x+\phi_{i, n}\right),
$$

where $\boldsymbol{k}_{n}$ is the wave vector of the KAW and $\omega_{\mathrm{KAW}}\left(\boldsymbol{k}_{n}\right)$ is the associated frequency for the propagation angles used in the present study (which vary between $\theta_{k B}=80^{\circ}$ and $\theta_{k B}=86^{\circ}$ ). This frequency is calculated numerically by solving the linearized FLR-LF equations, using the Maple software. The three considered models are numerically integrated using a Fourier spectral method in a 3D periodic domain. In the present simulations, the direction parallel to the ambient magnetic field is extended by a factor $\sim 6$, compared with the perpendicular direction in order to focus on the quasi-transverse dynamics. Weak hyperviscosity and hyperdiffusivity are supplemented to ensure the presence of a sufficient numerical dissipation, which were verified not to affect the spectral exponents. Two different scales of forcing corresponding to wavenumbers, $k_{\perp f} d_{i}=0.18$ (small scale) and $k_{\perp f} d_{i}=0.045$ (large scale), are considered. The FLR-LF simulations contain from $128^{3}$ for the small scale forcing to $512^{2} \times 256$ grid points for the larger scale forcing, before aliasing is removed. The associated spectral ranges are $0.18 \lesssim k_{\perp} \rho_{i} \lesssim 7.8$ and $0.045 \lesssim k_{\perp} \rho_{i} \lesssim 7.8$, respectively, after aliasing is removed, which in runs involving either large or small-scale forcing corresponds to a perpendicular mesh size of $\Delta x_{\perp}=0.27 d_{i}$. For CGL-HMHD runs with small-scale forcing, the spatial resolution is $256^{2} \times 512$, with a spectral range $0.18 \lesssim k_{\perp} \rho_{i} \lesssim 15.6$. For the CGL-HMHD runs with largescale forcing, the presence of waves and shocks requires a larger resolution in the parallel direction $\left(512^{2} \times 1024\right.$ grid points are used).

All the simulations presented here are done with plasma $\beta_{i}=1$, and initially equal and isotropic ion and electron temperatures $\left(d_{i}=\rho_{i}\right)$. The displayed spectra are shown as a function of $k_{\perp} d_{i}$ after integration on $k_{z} d_{i}$ and time averaging over a few outputs when the simulation reaches a quasistationary state.

In the following discussion, we show the results from various simulations using different levels of physics. This includes runs using the full FLR-LF code, the LF code (i.e., without FLR corrections), and the CGL-HMHD code (no FLRs and no Landau damping but with the Hall term in the Ohm's law). For all these runs, we investigate the role of the nonlinearity parameter (ratio, at a given wavenumber $k_{\perp}$, of the typical nonlinear frequency to the wave frequency $\chi \equiv \frac{\omega_{N L}}{\omega_{w}}$, which at MHD scales reduces to $\chi \equiv \frac{k_{\perp} \delta B_{\perp_{k}}}{k_{\|} B_{0}}$ ) on the scaling of the magnetic energy spectra at sub-ion scales. Furthermore, we separately consider the role of the amplitude fluctuation $\delta B_{\perp} / B$ 
Table 1

Driving Wavenumber $k_{f} d_{i}$, Amplitude Level $\delta B_{\perp} / B$, and Angle $\theta_{k B}$ Used for the FLR-LF, LF, and CGL-HMHD Runs

\begin{tabular}{llll}
\hline \hline Runs & $k_{f} d_{i}$ & $\delta B_{\perp} / B$ & $\theta_{k B}\left({ }^{\circ}\right)$ \\
\hline AA & 0.18 & 0.20 & 80 \\
A & 0.18 & 0.13 & 80 \\
B80 & 0.18 & 0.08 & 80 \\
B83 & 0.18 & 0.08 & 83.6 \\
B86 & 0.18 & 0.08 & 86 \\
LS1 & 0.045 & 0.13 & 83.6 \\
LS2 & 0.045 & 0.08 & 83.6 \\
\hline
\end{tabular}

and the wave vector anisotropy $k_{\perp} / k_{\|}$at the driving scale. The different parameters of the runs are summarized in Table 1.

\section{Energy Spectra and Dependence on the Forcing Amplitude}

\subsection{Numerical Simulations}

\subsubsection{Small-scale Driving}

In this section, we first explore the dependence of the spectra on the amplitude of the waves at the driving scale $k_{\perp f} d_{i}=0.18$ for a fixed propagation angle $\theta_{k B}=80^{\circ}$. We choose three different amplitude fluctuations $\delta B_{\perp} / B=0.2, \delta B_{\perp} / B=0.13$, and $\delta B_{\perp} / B=0.08$, where $\delta B_{\perp} / B$ stands for the time-averaged rms value of the dimensionless perpendicular magnetic component. For the two largest amplitudes, the corresponding values of the nonlinearity parameter of the FLR-LF runs are $\chi=0.30, \quad \chi=0.25$, while in the case of the smallest amplitude, the value of $\chi$ is smaller but not constant throughout the spectral domain, the regime being closer to weak turbulence (for details, see Sulem et al. 2016). The results are shown in Figure 1. The simulations show the development of a quasistationary turbulence energy cascade at scales $0.3 \lesssim k_{\perp} \rho_{i} \lesssim 8$. A first remark is that all the CGL-HMHD runs yield a powerlaw magnetic spectrum $E\left(B_{\perp}\right)$ with a slope close to $-7 / 3$, in some cases affected by small-scale bottleneck effects due to hyperdiffusivity. This result is consistent with theoretical predictions of turbulence based, for example, on incompressible Hall-MHD (Galtier 2008) and phenomenological arguments based on critical balance. This slope is similar to the one obtained from the LF and FLR-LF codes, when the fluctuation amplitude is the highest, $\delta B_{\perp} / B=0.2$ (Figure 1, top). The second remark is that, in contrast with the CGL-HMHD results, the FLR-LF and LF results show a dependence of the spectral slopes on the wave amplitude-the lower the amplitude, the steeper the spectrum. A phenomenological model of the energy cascade was developed to explain this result (Passot \& Sulem 2015; Sulem et al. 2016). The third interesting result is that the Landau damping alone leads to a significant steepening of the spectra at the kinetic scales. While Landau damping seems to actively participate in dissipating the magnetic energy, the FLR corrections appear to balance this effect by populating the spectrum with more power at high wavenumbers. This picture can be intuitively expected, considering that lower amplitude fluctuations imply weaker nonlinearities, and therefore a more prominent role of dissipation. It does not, however, reproduce solar wind observations, as will be discussed in Section 3.2.

The second set of runs consists in keeping the forcing amplitude constant $\left(\delta B_{\perp} / B=0.08\right)$ and varying the angle $\theta_{k B}$. The results are shown in Figure 1 (bottom) and Figure 2. The
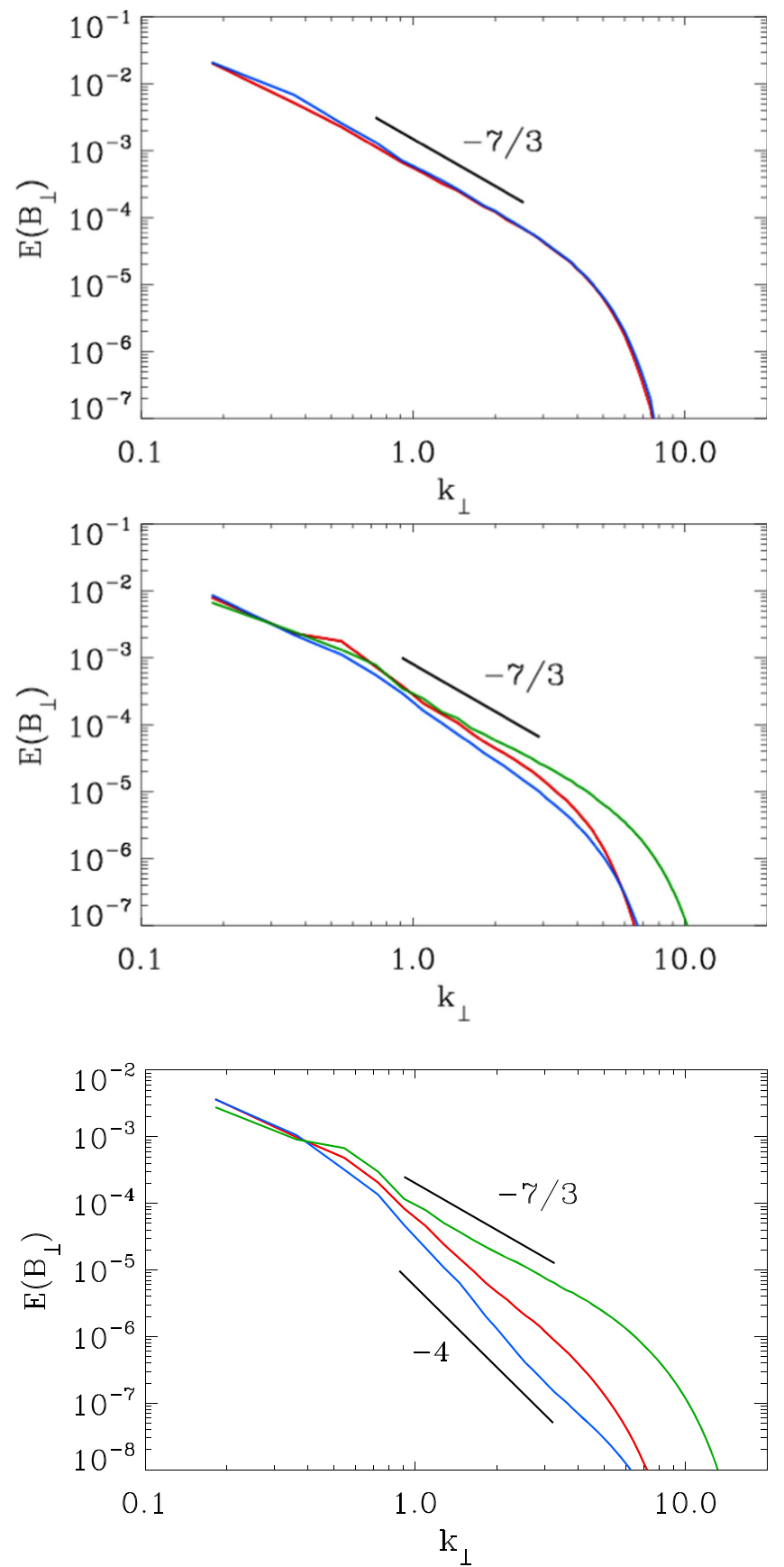

Figure 1. Magnetic energy spectrum $E\left(B_{\perp}\right)$ as a function of $k_{\perp}$ (in $d_{i}^{-1}$ units) for FLR-LF (red), LF (blue), and CGL-HMHD (green) models, with $k_{\perp f} d_{i}=0.18$ for different initial wave amplitude: $\delta B_{\perp} / B=0.2$ (top, run $\mathrm{AA}$ ), $\delta B_{\perp} / B=0.13$ (middle, run A), and $\delta B_{\perp} / B=0.08$ (bottom, run B80) and at a fixed propagation angle $\theta_{k B}=80^{\circ}$.

values of the nonlinearity parameter for the FLR-LF run with $\theta_{k B}=83^{\circ}$ (Figure 2, top) is $\chi=0.21$, while for the case $\theta_{k B}=86^{\circ}$ (Figure 2, bottom), it is $\chi=0.3$. Here, again, we find similar features as in the previous runs: the spectral slopes strongly depend on the propagation angles - the more oblique the waves at the driving scale, the shallower the spectrum. This result can be explained by similar intuitive arguments as noted previously: more oblique angles imply higher $k_{\perp} / k_{\|}$, which in turn implies a prominent role of the nonlinearities (i.e., larger values of $\chi$ ) over the dissipation. Note, however, that linear KAWs are weakly damped by the Landau resonance at high 

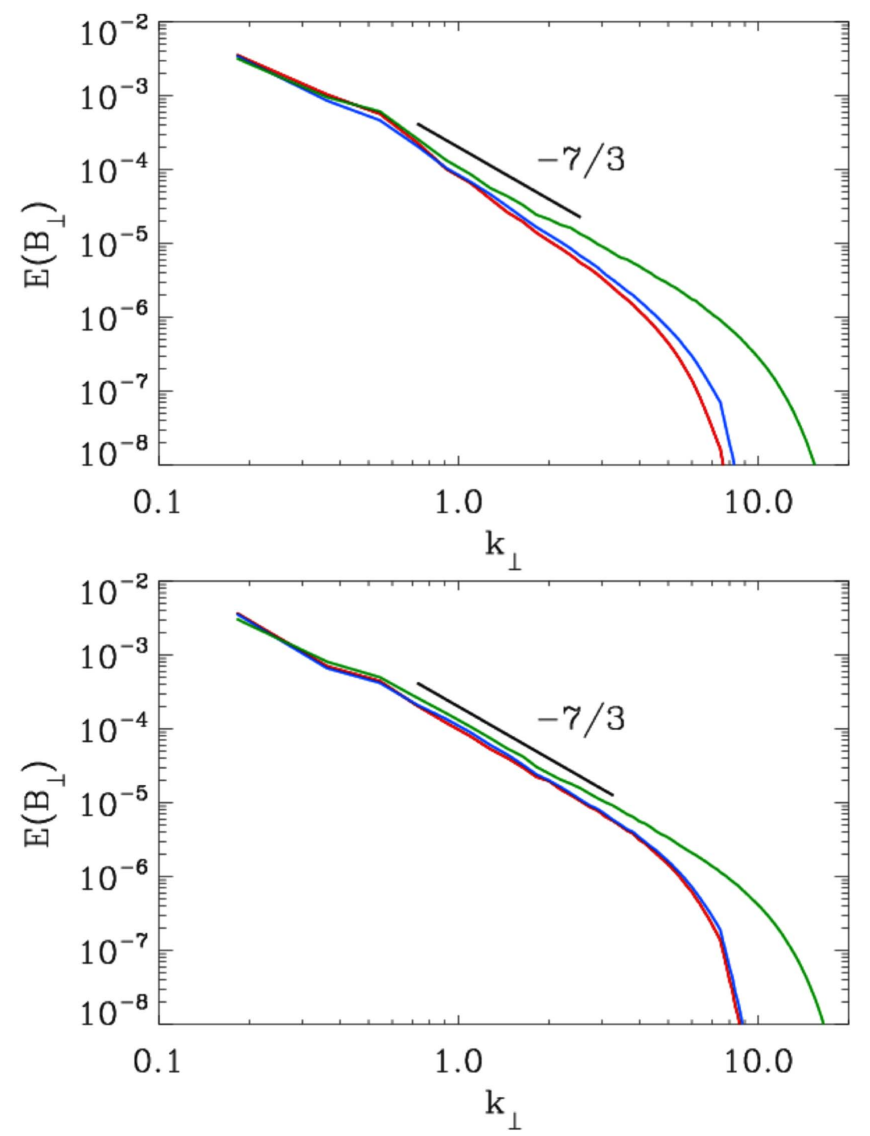

Figure 2. Magnetic energy spectrum $E\left(B_{\perp}\right)$ as a function of $k_{\perp}$ (in $d_{i}^{-1}$ units) for FLR-LF (red), LF (blue), and CGL-HMHD (green) models, with $k_{f} d_{i}=0.18$ for the propagation angles $\theta_{k B}=83^{\circ}$ (top, run B83) and $\theta_{k B}=86^{\circ}$ (bottom, run B86), and for a fixed wave amplitude $\delta B_{\perp} / B=0.08$. The case $\theta_{k B}=80^{\circ}$ is given in Figure 1 (bottom).

oblique angles than at moderate propagation angles (Sahraoui et al. 2012). These two effects (stronger nonlinearities and weaker Landau damping) may work together and lead to the observed shallower spectra at high oblique propagation angles.

\subsubsection{Large-scale Driving}

The results of the previous section may legitimately raise some concern about the proximity of the forcing scale $\left(k_{\perp_{f}} d_{i}=0.18\right)$ from the scales at which the turbulence spectra were obtained $\left(0.3 \lesssim k \rho_{i} \lesssim 8\right)$. The resulting lack of scale separation may cause the power-law spectra reported previously to be dependent on the nonlinearity parameter $\chi$. To test this hypothesis, we performed a new set of simulations where the driving scale is now shifted toward larger scales (i.e., to $\left.k_{\perp_{f}} d_{i}=0.045\right)$, which is four times larger than in the previous runs. We considered two cases with both the full FLRLF and the CGL-HMHD models. In the first one, the amplitude was fixed to $\delta B_{\perp} / B=0.08$ and $\theta_{k B}=83^{\circ} .6$. In the second case, we kept the same angle $\theta_{k B}$ but changed the amplitude to $\delta B_{\perp} / B=0.13$. The results are shown in Figures 3 and 4 for the FLR-LF and CGL-HMHD models, respectively. Interestingly, we observe that the two FLR-LF runs provide similar spectral slopes-close to $-5 / 3$ at large scales and -2.45 at small scales. For the CGL-HMHD model, in the large amplitude forcing case, a transition between the MHD range with a $-5 / 3$ magnetic spectrum and the sub-ion scales is still visible, but the
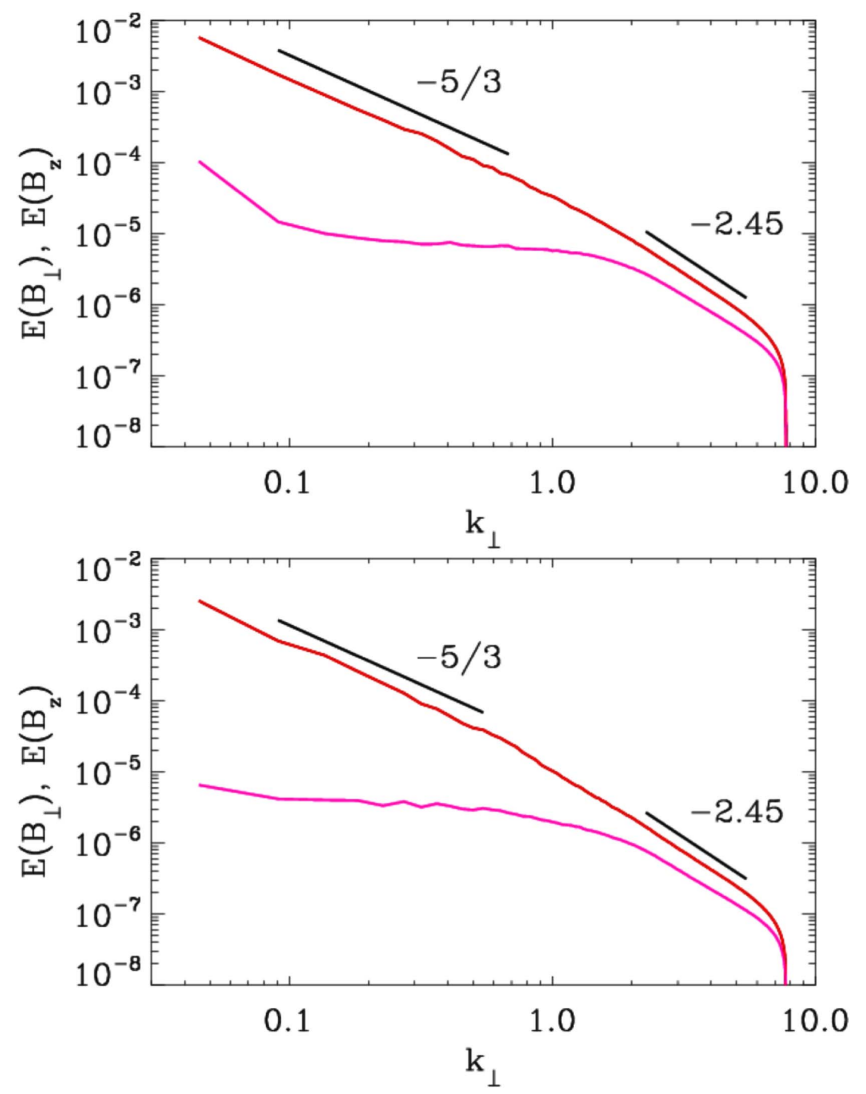

Figure 3. Perpendicular $E\left(B_{\perp}\right)$ (red) and parallel $E\left(B_{z}\right)$ (pink) magnetic energy spectrum as a function of $k_{\perp}$ (in $d_{i}^{-1}$ units) for the FLR-LF model for two different run with a driving at large scales: $\delta B_{\perp} / B=0.13$ and $\theta_{k B}=83^{\circ} .6$ (top) and $\delta B_{\perp} / B=0.08$ and $\theta_{k B}=83^{\circ} .6$ (bottom).

present resolution does not permit the observation of a clear power-law in the latter range. The presence of the large-scale shocks in this simulation indeed requires larger hyperviscosity and hyperdiffusivity (larger by a factor of 10 compared with the runs with Landau damping) at the present resolution. Note that such shocks do not develop when turbulence is driven at smaller scale, because of the enhanced dispersive effects. In CGL-HMHD simulations where the driving is weaker (Figure 4 , bottom), the $-5 / 3$ range is less visible, but a clear $-7 / 3$ sub-ion range is present. This suggests that this $-7 / 3$ spectrum is universal for the CGL-HMHD model. Another conspicuous difference between the FLR-LF and the CGLHMHD models concerns the $B_{z}$ spectrum $E\left(B_{z}\right)$ at large scale. While it is flat at a relatively low forcing amplitude in the FLRLF simulation (Figure 3) due to Landau damping, much more energy is carried by the largest scales for the CGL-HMHD model (Figure 4). The signature of this observation in physical space will be discussed in Section 4.

The behavior of the sub-ion range spectrum in the FLR-LF simulations thus contrasts with the results reported in the previous section: there is no clear slope variation when changing the amplitude, as was the case with forcing at small scales. Therefore the variation of the spectral slopes at the subion scales is due not only to the nonlinear parameter $\chi$ but also to the choice of the driving scale. The effect of the fluctuations at various scales on the spectral slopes can be directly verified in the solar wind using spacecraft observations, which we discuss in the following section. 

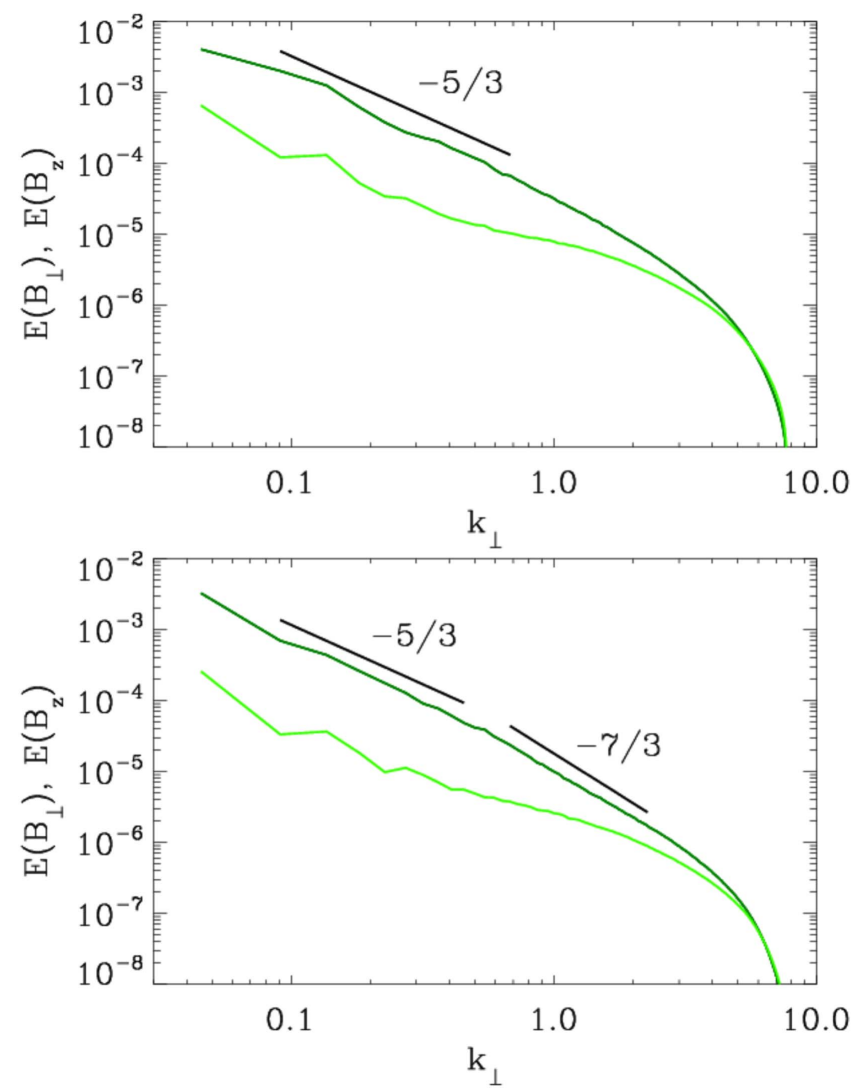

Figure 4. Perpendicular $E\left(B_{\perp}\right)$ (green) and parallel $E\left(B_{z}\right)$ (light green) magnetic energy spectrum as a function of $k_{\perp}$ (in $d_{i}^{-1}$ units) for the CGLHMHD model for two different runs with a driving at large scales: $\delta B_{\perp} / B=0.13$ and $\theta_{k B}=83^{\circ} .6$ (top), and $\delta B_{\perp} / B=0.08$ and $\theta_{k B}=83^{\circ} .6$ (bottom).

\subsection{Spacecraft Observations}

We performed a large survey of the power spectra of the magnetic fluctuations measured by the Cluster spacecraft in the free-streaming solar wind (i.e., not connected to the bow shock). We combined the data from the Fluxgate (FGM) and the Seach-Coil magnetometers (STAFF-SC) onboard Cluster 2 (merged at the frequency $\sim 1 \mathrm{~Hz}$; Sahraoui et al. 2010). The data allow one to cover the frequency range $\sim[0.001,10] \mathrm{Hz}$ corresponding to the MHD and sub-ion scales. When STAFF$\mathrm{SC}$ is in burst mode (BM), its waveforms are sampled at $450 \mathrm{~Hz}$, which allows for exploring higher frequencies that reach the electron scales (Kiyani et al. 2009; Sahraoui et al. 2009, 2010). Due to the limited available BM data in the solar wind (Sahraoui et al. 2013), and because we are interested in investigating scales comparable to the ion scale, we use essentially the Cluster data in normal mode (NM). This significantly increases the size of our statistical sample in comparison with that used in Sahraoui et al. (2013).

An example of the analyzed magnetic energy spectra is displayed in Figure 5, which shows three ranges of scales with differents spectral slopes: $f^{-1.64}$ at MHD scales, $f^{-3.58}$ in the transition range (near the ion characteristic scale), and $f^{-2.57}$ at the sub-ion scales. To compare the Cluster observations with the previous simulation results, we integrated the power of the fluctuations in different bandwidths (marked in Figure 5): $[0.01,0.1] \mathrm{Hz},[0.1,0.5] \mathrm{Hz}$, and $[1,2] \mathrm{Hz}$, which correspond respectively to the spatial scales $k \rho_{i} \sim[0.01,0.11], k \rho_{i} \sim$ $[0.11,0.5]$, and $k \rho_{i} \sim[1.1,2.1]$ (using the Taylor hypothesis
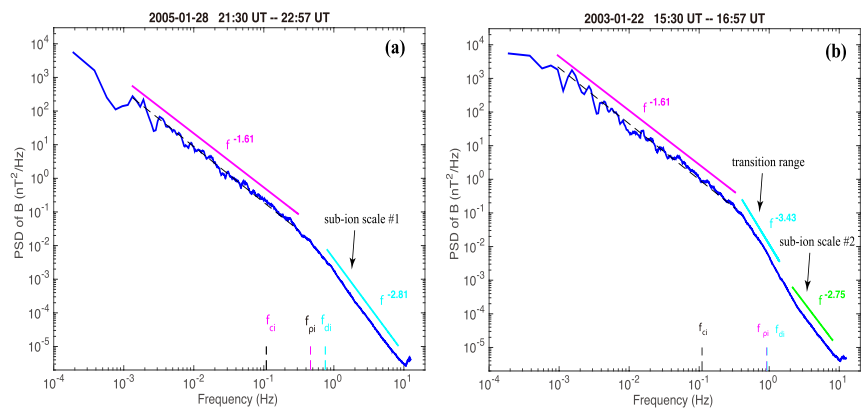

Figure 5. An example of power spectra of the magnetic fluctuations measured by the FGM $(f<1 \mathrm{~Hz})$ and STAFF-SC $(f>1 \mathrm{~Hz})$ experiments onboard Cluster 2. Vertical dashed lines are the proton gyrofrequency, $f_{c i}$, and the "Taylor-shifted" proton gyroradius and inertial length $\left(f_{\rho_{i}, d_{i}}=V_{f} / 2 \pi \rho_{i}, d_{i}\right)$.

and the average solar wind parameters: $V \sim 536 \mathrm{~km} \mathrm{~s}^{-1}$, $T \sim 22 \mathrm{eV}$ and $\left.n \sim 6 \mathrm{~cm}^{-3}, B \sim 7 \mathrm{nT}\right)$. The resulting normalized power $W / W_{o}$ ( $W_{o}$ is to the minimum observed integrated power for each bandwidth) is then correlated to the spectral slopes at the sub-ion scales. The results for the three bandwidths are shown in Figure 6.

Figures 6(a) and (b) depict a similar trend than that reported in Bruno et al. (2014) - the higher the power in the inertial range, the steeper the spectrum-although here we obtain a different nonlinear fit. This discrepancy may be due to the difference in the size of the statistical samples used in the two studies (here we used 760 intervals of $90 \mathrm{mn}$ duration each). Two more interesting features can be seen in Figure 6. First, one observes no correlation between the slopes in sub-ion scales 2 (blue circles) and the fluctuations amplitude calculated in the three frequency bandwidths. This result is consistent with our simulation results of Figure 3. Second, in Figure 6(c), for which the power has been integrated in the frequency range closest to the transition range, there is no clear correlation between the spectral slopes and the fluctuations power at the intermediate scales. This observation does not fully explain our simulations results, since we do not observe the same trend in the simulations and the spacecraft observations (i.e., the simulations show that high amplitude fluctuations yield shallower spectra at sub-ion scales). Nevertheless, it does show that what seems to influence the spectral slopes in the transition range is the fluctuations power in the inertial range. To prove that in the numerical simulations would require using a broader range of the fluctuations amplitude $\delta B_{\perp} / B$ than that used in the present simulations, and a broader dynamical range to possibly evidence the transition range. However, the physics of the transition range being poorly understood, input from spacecraft observations will be needed to define appropriate simulation setups and physical conditions to run such simulations.

\section{Nature of the Turbulent Fluctuations}

The partition of the turbulent energy transferred from the inertial range into ion and electron heat is an important unresolved problem, because it is strongly related to which species the energy ends up being and how this transition occurs. Figures 7(a)-(d) show the perpendicular magnetic energy as a function of $\omega$ and $|k|$ for run A with different angles $\theta_{k B}$. The FLR-LF dispersion relations are over-plotted for KAW (dashed blue), slow (dashed-dotted purple), and fast (three dots-dashed red) modes. We observe a maximum of energy at 

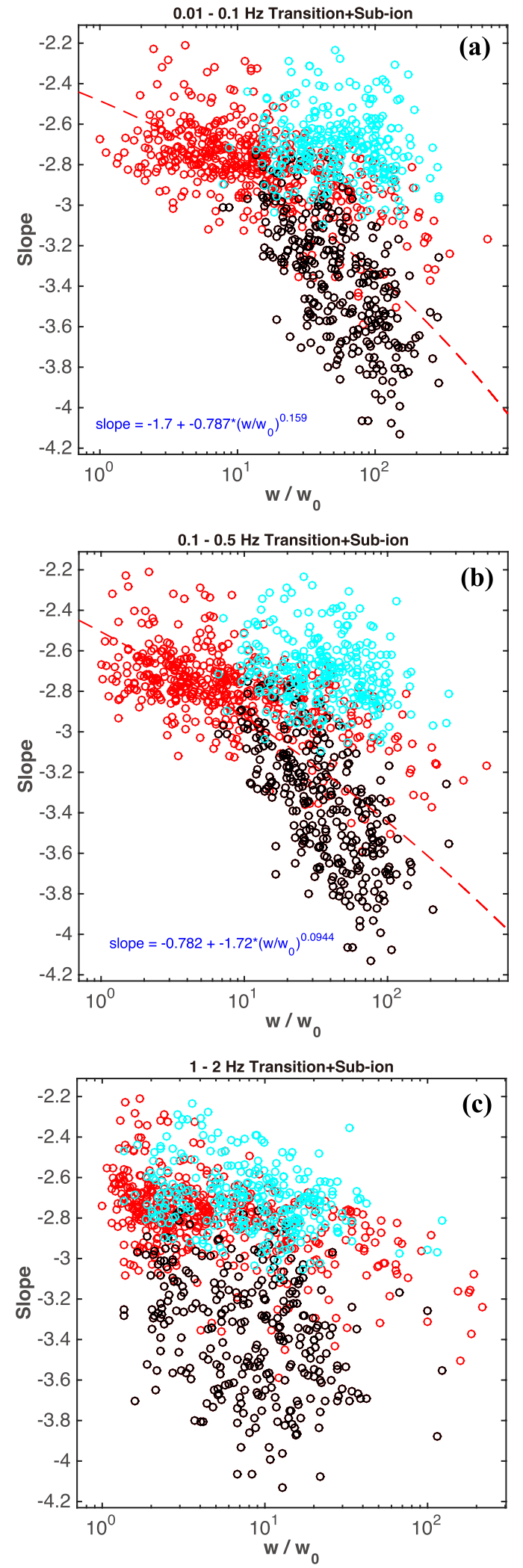

Figure 6. Cluster observations in the solar wind showing the correlation between the amplitude of the turbulent fluctuations measured within three bandwidths, [0.0, 0.1] $\mathrm{Hz}(\mathrm{a}),[0.1,0.5] \mathrm{Hz}(\mathrm{b})$, and [1, 2] $\mathrm{Hz}$ (c), with the spectral slopes in the transition range (black), in the sub-ion scale 1 (red), and in the sub-ion scale 2 (blue). See the definition in Figure 5.

the smallest $k$ and lowest $\omega$ region in Figure 7(c), which corresponds to the injected KAWs (at a $80^{\circ}$ angle). While the results indicate that the energy is mostly distributed at higher propagation angles, which is consistent with the theory of KAW turbulence, one finds a clear signature of the generation of the fast waves in KAW-driven turbulence with decreasing $\theta_{k B}$. The fast wave energy starts to be non-negligible at intermediate angles around $\theta \sim 60^{\circ}$ and below (Figure 7(a)). Nevertheless, turbulence energy is distributed mostly in KAW/ slow-mode regions (the energy distribution around the fast wave branch being much smaller, by a factor $\times 10^{-1}$ or less, compared with the $\mathrm{KAW} /$ slow counterparts at the ion gyroscale). Noticeably, the energy distribution around the $\mathrm{KAW} /$ slow modes is very broad, indicating strong nonlinearities associated with the presence of coherent structures (discussed later).

To further investigate the nature of the sub-ion scale turbulence, we use the magnetic compressibility defined, for each wavenumber $k_{\perp}$, as $C_{\|}=\delta B_{\|}^{2} /\left(\delta B_{\|}^{2}+\delta B_{\perp}^{2}\right)$, where $\delta B_{\|}$ and $\delta B_{\perp}$ are the parallel and perpendicular magnetic Fourier modes. It is computed for different runs from Table 1 and compared with the linear Vlasov-Maxwell solution for KAW, obtained using the WHAMP code $\left(B \sim 4.5 \mathrm{nT}, \beta_{i} \sim 1.5\right.$, $\beta_{e} \sim 1.2, \theta_{k B}=85^{\circ}$ ) and to Cluster observations in the solar wind used in Sahraoui et al. (2010). The results are shown in Figure 8. The three models, FLR-LF (red), the LF (blue), and the CGL-HMHD (green), show a magnetic compressibility increasing with $k_{\perp}$ and reaching isotropy (light black dotted line, $C_{\|}=1 / 3$ ) around the ion scale. This result is in general agreement with solar wind observations (black dotted line). However, at the sub-ion scales, the spacecraft observations are better reproduced by the FLR-LF results, while the CGLHMHD ones fit better $C_{\|}$at large scales. This is probably related to the fact that in the simulations, energy is injected in the form of pure Alfvénic fluctuations, whereas in the solar wind, a small fraction of compressible (fast or slow) waves can be present at large scales.

Another interesting question to investigate is the influence of Landau damping and FLR corrections on the magnetic compressibility by comparing FLR-LF and CGL-HMHD simulations. Figure 8 shows that for the FLR-LF simulations (red lines), $C_{\|}$is small at large scales and approaches equipartition at a scale slightly smaller than the ion Larmor radius, as predicted by linear theory, while in the CGL-HMHD runs (green lines), the compressibility is already significant at the largest scales. This effect is possibly due to the presence of fast waves, for which the linear theory predicts that the magnetic compressibility is close to unity at large scales (see, e.g., Figure 4 of Hadid et al. 2015). The slow increase of $C_{\|}$ visible for $k_{\perp} d_{i}>5$ in the case of the FLR-LF simulation is probably due to the hyperdiffusivity. The continuous growth observed in the case of the CGL-HMHD simulation seems, however, to be physical. By comparing LF (blue) and FLR-LF (red) simulations, a clear difference is visible at large scales, where the presence of FLR corrections make the magnetic compressibility significantly closer to the prediction of the linear kinetic theory. Furthermore, while for the CGL-HMHD description the driving scale has no conspicuous effect on $C_{\|}$ (dotted or solid green lines), we note that in the case of the FLR-LF model, we observed an enhanced magnetic compressibility at intermediate scales for the small-scale driving (dotted or solid red lines).

The differences between the two families of simulations are also visible in physical space (Figure 9). Indeed, parallel and perpendicular sections for the parallel magnetic fluctuations $B_{z}$ 
(a)

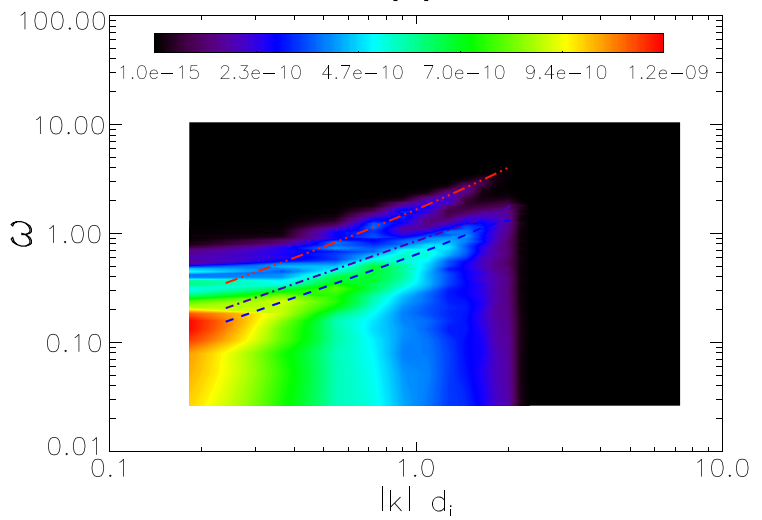

(c)

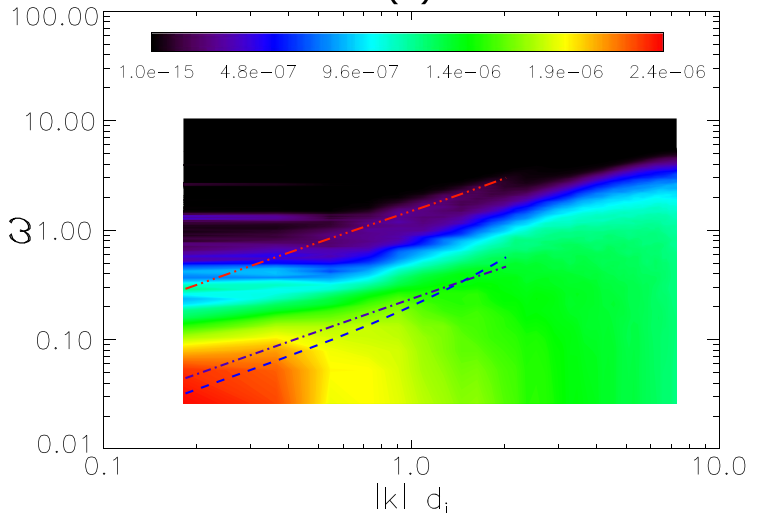

(b)

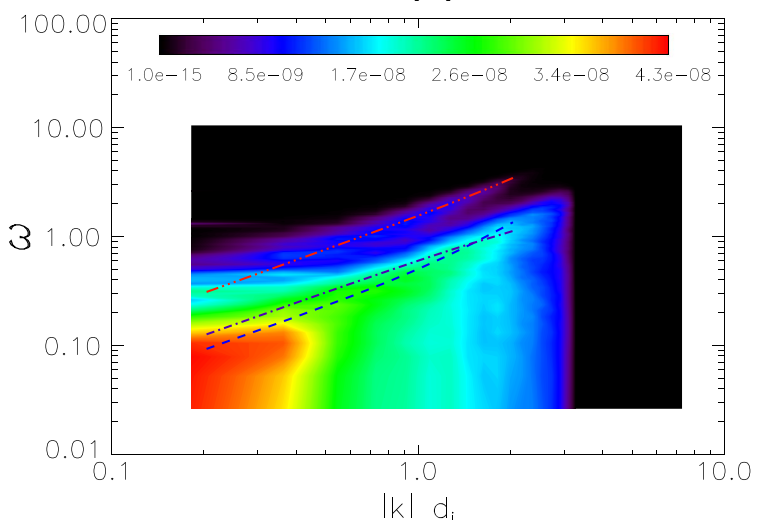

(d)

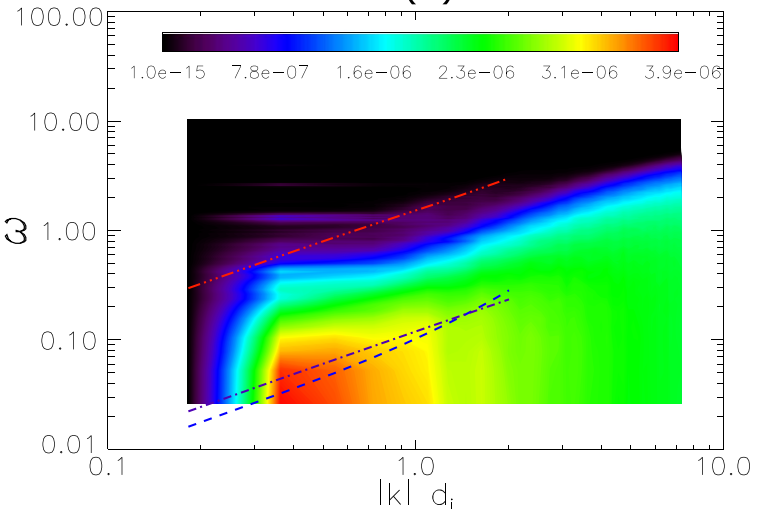

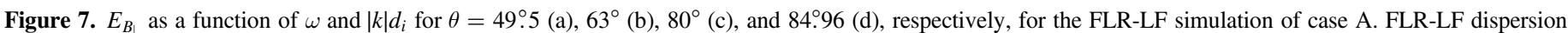
relations are over-plotted for KAW (dashed blue), slow (dashed-dotted purple), and fast (three dots-dashed red) modes.

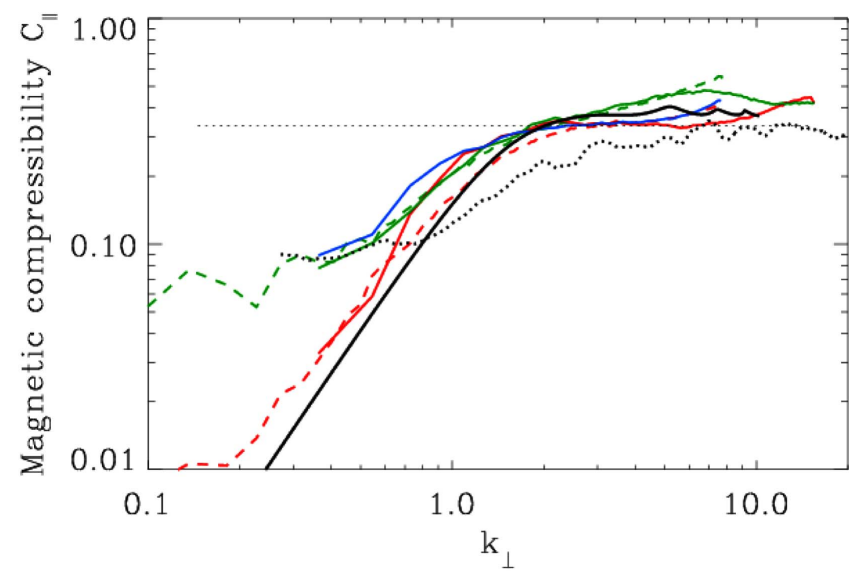

Figure 8. Magnetic compressibility $C_{\|}$as a function of $k_{\perp}$ (in $d_{i}^{-1}$ units) for simulations LS2 of the FLR-LF model (red dashed) and the CGL-HMHD ones (green dashed), and for run B3 in the FLR-LF (red solid), the LF (blue solid), and the CGL-HMHD versions (green solid). FLR-LF simulations are consistent with Vlasov theory (black solid). Cluster observations in the solar wind (201001-10, 06h15-06h25) are also displayed (black dots). In all cases, isotropy (light black dotted) is reached at small scales.

show the presence of short-scale waves and large-scale shocks, only in the case of CGL-HMHD simulations. They are not visible in the corresponding cross sections for FLR-LF simulations, due to the action of Landau damping. In both cases, we note the presence of sheets and filaments elongated along the magnetic field lines. Nevertheless, the filaments are less space-filling and rather isolated when compared with the CGL-HMHD simulation, where fluctuations are fuzzier and piled up behind shock waves. This observation is related to the presence of relatively strong energy at large scale in the $B_{z}$ spectrum (see Figure 4). More fragmentation of the structures is also observed in the case of the FLR-LF simulations because of FLR effects. Confirmation of the coexistence of sheet-like and filamentary structures is also seen at the level of the current density, as shown for the FLR-LF simulation in Figure 10. Density structures, also in the form of sheets, together with quasi-planar ion velocity streamlines, perpendicular to the ambient magnetic field, are shown in Figure 11. The ion flow is quasi-two-dimensional and is only weakly compressible.

\section{Discussion}

This paper aimed to identify the effects of Landau damping and FLR corrections on the dynamics of three-dimensional Alfvén wave turbulence, with a special focus on the sub-ion scales. The study is performed by using numerical simulations of three different models, namely CGL-HMHD, LF, and FLRLF. The main observations concern the $-7 / 3$ universal CGLHMHD sub-ion spectrum of the transverse magnetic fluctuations, which contrasts with both the LF and FLR-LF spectra, whose slopes are observed to depend on the nonlinearity parameter, and on the driving scale. This non-universality of the spectral exponent is shown to be associated with Landau damping. Note that FLR corrections have a tendency to enhance the small-scale nonlinearities. They also ensure a better fit of the magnetic compressibility with the prediction of the linear kinetic theory. A question arises about the correlation between the amplitude of the magnetic fluctuations and the 

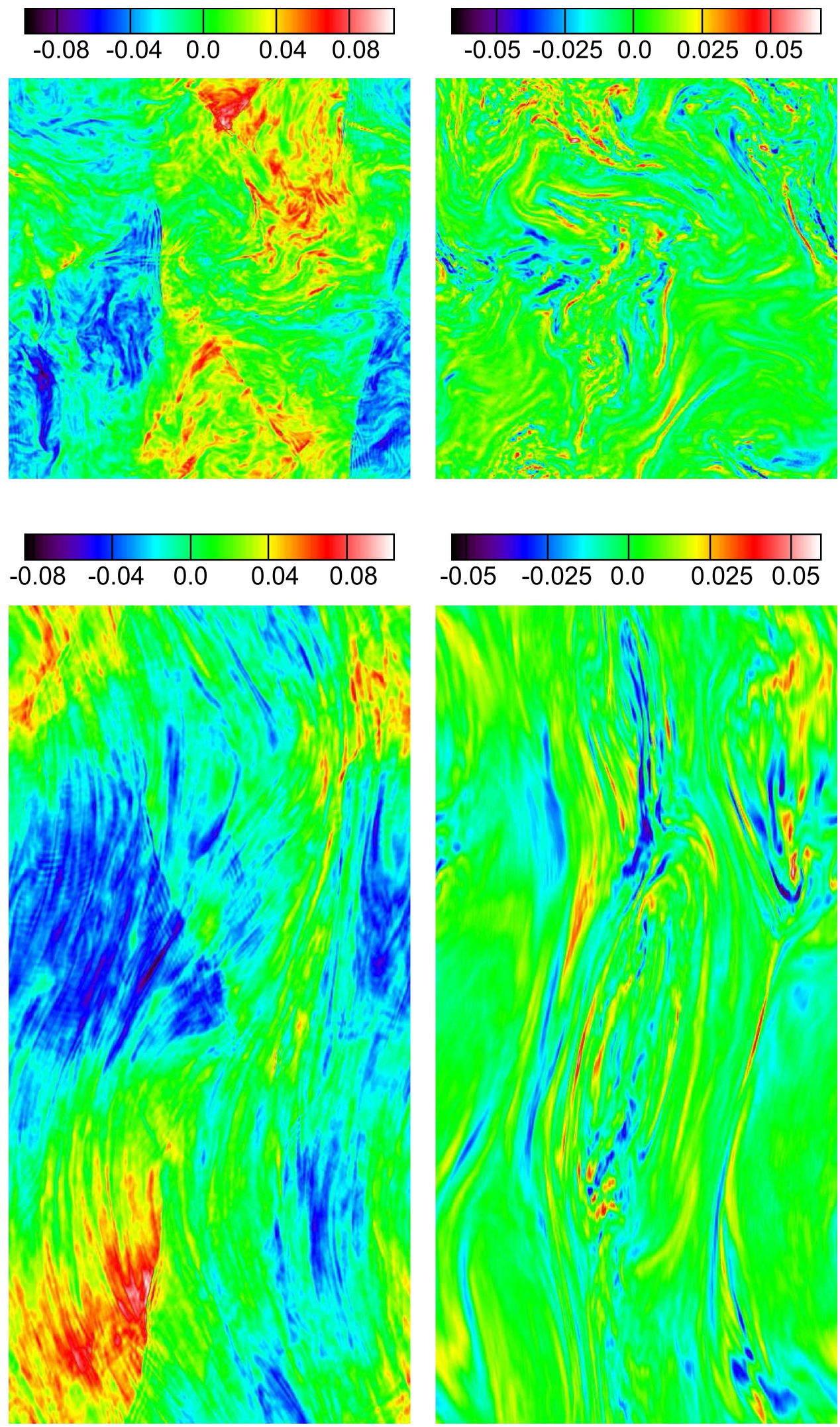

Figure 9. Transverse (top) and longitudinal (bottom) sections of the $B_{z}$ field for the CGL-HMHD (left) and FLR-LF (right) simulations in case LS2. Note the presence in the CGL-HMHD simulation of short-scales waves mostly visible within the (blue) structures in the middle left part of the longitudinal section. Shocks are conspicuous on both longitudinal and transverse sections.

slope of the magnetic exponent in the sub-ion range. At this level, the comparison with spacecraft observations is delicate. First, from the theoretical side, the control parameter (i.e., the nonlinearity parameter) is a combination of amplitude and typical propagation angle, which is difficult to evaluate accurately from spacecraft data (Sahraoui et al. 2010). Second, 


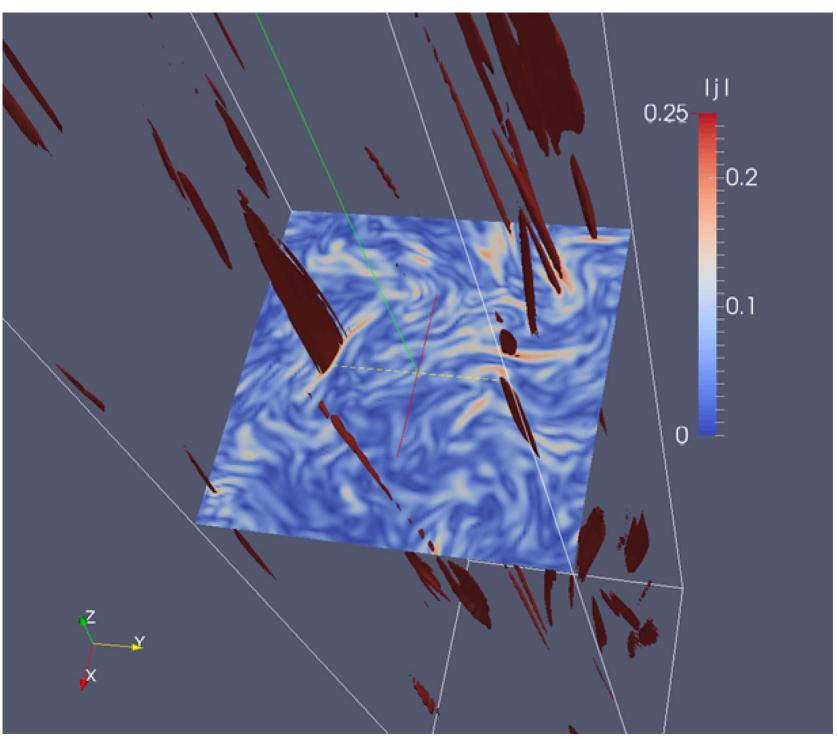

Figure 10. FLR-Landau fluid simulation demonstrates the coexistence of sheelike and filamentary structures of current density $J$.

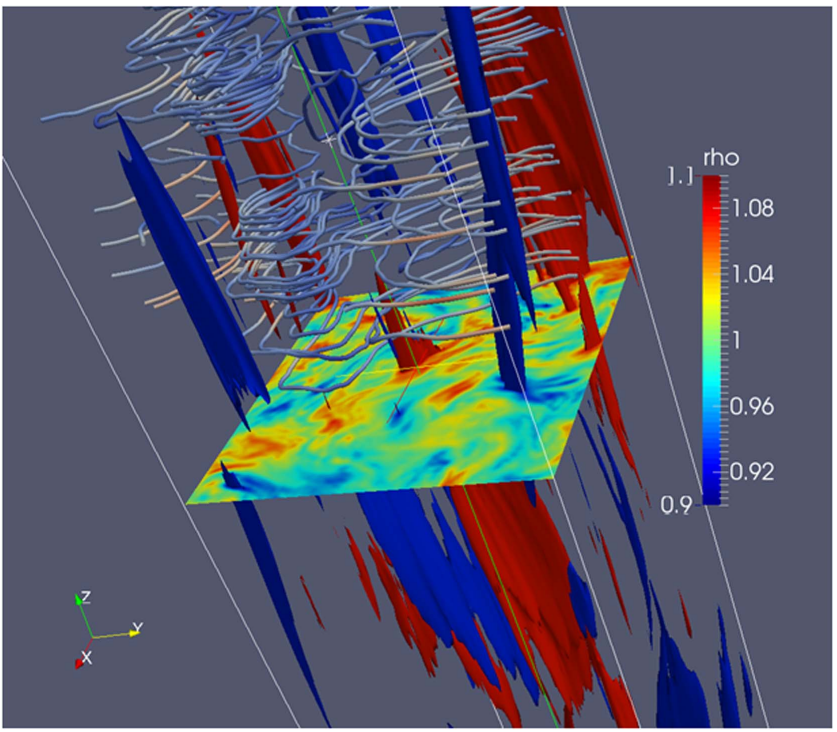

Figure 11. FLR-Landau fluid simulation showing typical density isosurfaces with ion velocity streamlines. The latter mostly lie in planes perpendicular to the ambient magnetic field.

the observed spectrum in spacecraft data can display a much steeper transition range (not present in simulations), whose physics is still poorly understood. This led us to perform a new analysis, using data measured by the Cluster spacecraft in the solar wind, that covered several spectral ranges where wave amplitude and spectral slopes are evaluated. When correlating the wave amplitude measured in the inertial range with slopes measured in all the ranges, including the transition one, we found a tendency for steeper spectra at larger amplitudes, as in Bruno et al. (2014). This correlation is the opposite of the one found in simulations driven at scales close to the ion Larmor radius. On the contrary, when observational slopes in the subion range, excluding the transition one, are correlated with amplitudes measured closer to the ion Larmor radius, no clear correlation is found. When the slopes are measured further down in the sub-ion range (when a transition range is not present at the ion scales), no correlation is found with the fluctuation amplitude, regardless of the frequency bandwidth where the amplitude measurement is performed. This observation can be linked to numerical simulation results with a forcing at large scale (i.e., that included part of the MHD range), which showed no evidence for a change of slope when varying the driving amplitude.

Numerical simulations also enable one to investigate the geometrical properties of the flow structures and the type of waves that are present. Noticibly, in contrast with the CGLHMHD model, the FLR-LF one correctly reproduces the variation with the transverse wavenumber of the observational magnetic compressibility. Furthermore, the CGL-HMHD simulations show the presence of small-scale waves and large-scale shocks that contribute to structure the flow. These fast waves are mostly damped in the FLR-LF simulations, and are only visible at some oblique angles, as revealed by an analysis of the frequency-wavenumber spectrum. In the FLRLF simulations, thin and rather isolated filamentary structures aligned with the local magnetic field coexist with sheets.

This study demonstrates the need for Landau damping in realistic simulations of the solar wind. More precise comparisons with spacecraft observations nevertheless require higher resolution simulations, as the turbulence properties are very sensitive to the type and spectral location of the driving process. This type of study is planned together with a more detailed analysis of the energy distribution among the various types of waves and coherent structures.

We thank G. Howes for fruitful discussions. This work was granted access to the HPC resources oF CINES/IDRIs under the allocation 2016047042. Part of the computations have been done on the "Mesocentre SIGAMM" machine, hosted by Observatoire de la Côte d'Azur. S.K. is funded by the ANR project THESOW, grant ANR-11-JS56-0008. The French participation in the Cluster project is funded by CNES.

\section{References}

Alexandrova, O., Carbone, V., Veltri, P., \& Sorriso-Valvo, L. 2008, ApJL, 674,1153

Alexandrova, O., Lacombe, C., Mangeney, A., Grappin, R., \& Maksimovic, M. 2012, ApJ, 760, 121

Bale, S. D., Kasper, J. C., Howes, G. G., et al. 2009, PhRvL, 103, 21101

Bale, S. D., Kellogg, P. J., Mozer, F. S., Horbury, T. S., \& Rème, H. 2005, PhRvL, 94, 215002

Banerjee, S., Hadid, L. Z., Sahraoui, F., \& Galtier, S. 2016, ApJL, 829, L27

Bruno, R., \& Carbone, V. 2005, LRSP, 2, 4

Bruno, R., Trenchi, L., \& Telloni, D. 2014, ApJL, 793, L15

Camporeale, E., \& Burgess, D. 2011, ApJ, 730, 114

Carbone, V., Marino, R., Sorriso-Valvo, L., Noullez, A., \& Bruno, R. 2009 PhRvL, 103, 061102

Chang, O., Peter Gary, S., \& Wang, J. 2011, GeoRL, 38, L22102

Chasapis, A., Retino, A., Sahraoui, F., et al. 2015, ApJL, 804, L1

Chen, C. H. K., Boldyrev, S., Xia, Q., \& Perez, J. C. 2013, PhRvL, 110, 225002

Chew, G. F., Goldberger, M. L., \& Low, F. E. 1956, RSPSA, 236, 112

Galtier, S. 2008, PhRvE, 77, 015302

Gary, S. P., Chang, O., \& Wang, J. 2012, ApJ, 755, 142

Gary, S. P., Fuselier, S. A., \& Anderson, B. J. 1993, JGR, 98, 1481

Goldstein, M. L., Roberts, D. A., \& Fitch, C. A. 1994, JGR, 99, 11519

Grison, B., Sahraoui, F., Lavraud, B., et al. 2005, AnGeo, 23, 3699

Hadid, L. Z., Sahraoui, F., \& Galtier, S. 2017, ApJ, 838, 9

Hadid, L. Z., Sahraoui, F., Kiyani, K., et al. 2015, ApJL, 813, L29

He, J., Marsch, E., Tu, C., Yao, S., \& Tian, H. 2011, ApJL, 731, 85

Hellinger, P., Trávníček, P., Kasper, J. C., \& Lazarus, A. J. 2006, GeoRL, 33, L09101

Howes, G. G. 2015, JPIPh, 81, 325810203

Howes, G. G., Bale, S. D., Klein, K. G., et al. 2012, ApJL, 753, L19

Howes, G. G., Dorland, W., Cowley, S. C., et al. 2008, PhRvL, 100, 065004 
Howes, G. G., TenBarge, J. M., Dorland, W., et al. 2011, PhRvL, 107, 035004 Huang, S. Y., Hadid, L., Sahraoui, F., Yuan, Z. G., \& Deng, X. H. 2017a, ApJL, 836, L10

Huang, S. Y., Sahraoui, F., Deng, X. H., et al. 2014, ApJL, 789, L28

Huang, S. Y., Sahraoui, F., Retino, A., et al. 2016, GeoRL, 43, 7850

Huang, S. Y., Sahraoui, F., Yuan, Z. G., et al. 2017b, ApJL, 836, L27

Hunana, P., Goldstein, M. L., Passot, T., et al. 2013, ApJ, 766, 93

Hunana, P., Laveder, D., Passot, T., Sulem, P. L., \& Borgogno, D. 2011, ApJ, 743,128

Karimabadi, H., Roytershteyn, V., Wan, M., et al. 2013, PhPl, 20, 012303

Kasper, J. C., Lazarus, A. J., \& Gary, S. P. 2002, GeoRL, 29, 20

Kiyani, K. H., Chapman, S. C., Khotyaintsev, Y. V., Dunlop, M., \& Sahraoui, F. 2009, PhRvL, 103, 075006

Kiyani, K. H., Chapman, S. C., Sahraoui, F., et al. 2013, ApJ, 763, 10

Klein, K. G., Howes, G. G., TenBarge, J. M., \& Podesta, J. J. 2014, ApJ, 785, 138

Leamon, R. J., Matthaeus, W. H., Smith, C. W., \& Wong, H. K. 1998a, ApJL, 507, L181

Leamon, R. J., Smith, C. W., Ness, N. F., \& Matthaeus, W. H. 1998b, JGR, 103,4775

Leamon, R. J., Smith, C. W., Ness, N. F., \& Wong, H. K. 1999, JGR, 104, 22331

Markovskii, S. A., \& Vasquez, B. J. 2011, ApJ, 739, 22

Marsch, E. 2006, LRSP, 3, 1

Marsch, E., Ao, X.-Z., \& Tu, C.-Y. 2004, JGR, 109, A04102

Matteini, L., Landi, S., Hellinger, P., et al. 2007, GeoRL, 34, L20105

Meyrand, R., \& Galtier, S. 2012, PhRvL, 109, 194501

Parashar, T. N., Servidio, S., Breech, B., Shay, M. A., \& Matthaeus, W. H. 2010, PhPl, 17, 102304
Passot, T., Henri, P., Laveder, D., \& Sulem, P. L. 2014, EPJD, 68, 207

Passot, T., \& Sulem, P. L. 2007, PhPl, 14, 082502

Passot, T., \& Sulem, P. L. 2015, ApJL, 812, L37

Passot, T., Sulem, P. L., \& Hunana, P. 2012, PhPl, 19, 082113

Plunk, G., Cowley, S., Shekochihin, A., \& Tatsuno, T. 2010, JFM, 664, 407

Podesta, J. J., \& Gary, S. P. 2011, ApJ, 742, 41

Podesta, J. J., \& TenBarge, J. M. 2012, JGR, 117, A10106

Sahraoui, F., Belmont, G., \& Goldstein, M. L. 2012, ApJ, 748, 100

Sahraoui, F., Belmont, G., Pinçon, J. L., et al. 2004, AnGeo, 22, 2283

Sahraoui, F., Belmont, G., Rezeau, L., et al. 2006, PhRvL, 96, 7

Sahraoui, F., Goldstein, M. L., Belmont, G., Canu, P., \& Rezeau, L. 2010, PhRvL, 105, 131101

Sahraoui, F., Goldstein, M. L., Robert, P., \& Khotyaintsev, Y. V. 2009, PhRvL, 102, 231102

Sahraoui, F., Huang, S. Y., Belmont, G., et al. 2013, ApJ, 777, 15

Sahraoui, F., Pincon, J. L., Belmont, G., et al. 2003, JGRA, 108, 1335

Schekochihin, A. A., Cowley, S. C., Dorland, W., et al. 2009, ApJS, 182, 310

Smith, C. W., Hamilton, K., \& Vasquez, B. J. 2006, ApJ, 645, L85

Snyder, P. B., Hammett, G. W., \& Dorland, W. 1997, PhPl, 4, 3974

Sulem, P. L., \& Passot, T. 2015, JPIPh, 81, 325810103

Sulem, P. L., Passot, T., Laveder, D., \& Borgogno, D. 2016, ApJ, 818, 66

Tassi, E., Sulem, P. L., \& Passot, T. 2016, JPIPh, 82, 705820601

Tatsuno, T., Dorland, W., Shekochihin, A., et al. 2009, PhRvL, 103, 015003

TenBarge, J. M., \& Howes, G. G. 2013, ApJL, 771, L27

Told, D., Jenko, F., TenBarge, J. M., Howes, G. G., \& Hammett, G. W. 2015 , PhRvL, 115, 025003 